\title{
EL FLORECIMIENTO DE LAS ZONAS MARINAS ESPECIALMENTE SENSIBLES. ¿HACIA UNA NUEVA "BATALLA LIBRESCA" EN PLENO SIGLO XXI?
}

\section{David ENRÍQUEZ*}

RESUMEN: La construcción del principio de la libertad de navegación ha sido una tarea de siglos del derecho internacional del mar. Sin embargo, su consagración mediante la Convención de las Naciones Unidas sobre el Derecho del Mar de 1982 no es el último capítulo que sobre el tema se ha escrito. En efecto, el florecimiento de Zonas Marinas Especialmente Sensibles (ZMES) designadas recientemente por la Organización Marítima Internacional, impone límites de orden público internacional a la libertad de la navegación a favor de la protección del ambiente marino. El objeto del presente artículo es estudiar la problemática actual para encontrar un adecuado equilibrio entre libertad de navegación y protección del ambiente marino, mediante el hilo conductor de las Zonas Marinas Especialmente Sensibles y el polémico caso de dichas zonas en Europa Occidental.

ABSTRACT: The creation of the principle of free navigation has been an old task to the International Law of the Sea. However, its consecration through the 1982 UN Convention on the Law of the Sea is not the last chapter written about the theme. Certainly, the emergence of the Specially Sensitive Maritime Zones (SSMZ) so called recently by the International Maritime Association, sets international public limits to the free navigation in favor of the protection of the maritime environment. The purpose of this article is to analyse the current problem relating the quest of an adequate balance between free navigation and maritime environment protection, through the conducting line of the SSMZ and the polemic case of the Western European's SSMZs.

RÉSUMÉ: La construction du principe de la liberté de navégation a été une tâche séculaire pour le Droit International du Mer. Néanmoins, sa consacration à travers de la Convention des Nations Unies sur le Droit du Mer de 1982, n'est pas le dernier chapitre écrit sur ce thème. En effet, le développement des Zones Maritimes Spécialement Sensibles (ZMSS) récemment designées par l'Organisation Maritime International, impose des limites d'ordre publiques internationales à la libérté de navegation en faveur de la protection de l'environnement maritime. L'objectif de cet article c'est d'analyser la problématique actuelle a fin de trouver un équilibre approprié entre la liberté de navégation et la protection de l'environnement maritime, tout en observant les ZMSS et le cas très polémique des ZMSS de l'Europe de l'Ouest.

* Doctor en derecho, investigador nacional nivel 1 (SNI) e investigador titular del Instituto Panamericano de Jurisprudencia (UP). Representante del gobierno mexicano (SCT) ante la OMI, el FIDAC, la OIT, la CNUDMI, la OMC, la OCDE y la APEC, entre otros organismos internacionales. 
SUMARIO: I. Aproximación. II. La libertad de navegación. III. Las Zonas Marinas Especialmente Sensibles. IV. El caso de las ZMES de Europa Occidental. V. Conclusiones. Hacia una revisión de las Directrices sobre ZMES.

\section{APROXIMACIÓN}

Como se analizará a lo largo del presente artículo, tanto las Zonas Marinas Especiales (ZME) como las Zonas Marinas Especialmente Sensibles (ZMES) son áreas que por sus características representan limitaciones a la libertad de navegación, tendentes a la protección del medio ambiente marino.

Las dos figuras aludidas - libertad de navegación y zonas marinas de ambas categorías - cuentan con un sólido fundamento en las fuentes formales del derecho del mar y en el derecho marítimo respectivamente. ${ }^{1}$ Sin embargo, la reciente proliferación de solicitudes de ZMES a la Organización Marítima Internacional (OMI) por diversos países y grupos de países, no debe impedir hacer un alto en el camino, y reflexionar tanto sobre la justificación de algunas de las solicitudes, como sobre la posible necesidad de revisar los instrumentos internacionales aplicables. ${ }^{2}$

Tenemos, pues, dos bienes jurídicos tutelados - la libre navegación internacional y la protección de la biodiversidad marina- y un importante reto por lograr: la armonización de ambos, de modo que la designación de toda ZMES no represente un retroceso a la legítima libertad de navegación; una de las primordiales conquistas del derecho del mar, lograda tras siglos de guerras, debates y negociaciones internacionales. ${ }^{3}$

1 Véase Convención de las Naciones Unidas sobre el Derecho del Mar de 1982 (Convemar), artículos 192-194 y 211 (6); Convenio Internacional para Prevenir la Contaminación por los Buques de 1973, modificado por el Protocolo de 1978 (MARPOL 73/78), anexos I, II y V, Resolución A.927 (22) de la Asamblea de la OMI.

2 Véase Documento OMI, MEPC 51/WP.9 y MEPC 49/22.

3 Para una introducción al estudio del derecho del mar; véase, entre otros, Cervera, José, El derecho del mar, Madrid, Editorial Naval, 1992, pp. 37 y ss.; Gómez-Robledo, Alonso, Derecho del mar, México, UNAM, Instituto de Investigaciones Jurídicas, pp. 105 y ss.; Székely, Alberto, Derecho del mar, México, UNAM, Instituto de Investigaciones Jurídicas, 1991, pp. 27 y ss.; Scovazzi, Tullio, Elementos de derecho internacional del mar, Madrid, Tecnos, 1994, pp. 55 y ss.; Sobarzo, Alejandro, Régimen jurídico del alta mar, México, Porrúa, 1985, pp. 55 y ss.; Castañeda, Jorge, Obras completas. Derecho del mar, México, IMRED, 1995, pp. 101 y ss.; Enríquez, David, Historia del derecho maritimo mexicano, Colima, Gobierno del Estado de Colima, 1997, pp. 2 y ss. 
En este contexto, el objeto del presente estudio es analizar el debate que hasta la actual fecha diversos Estados miembros de la OMI sostienen respecto a la licitud de diversas ZMES recientemente designadas o en trámite de designación, y a partir de ello describir brevemente las tendencias revisionistas que sobre el tema podrían existir en el mediato plazo.

Con el fin de ofrecer una exposición ordenada de la discusión, se ha dividido el contenido en cinco epígrafes a lo largo de los cuales se pretende aludir a la libertad de navegación en el derecho del mar, a los instrumentos internacionales aplicables en la designación de ZMES, a la confrontación generada en el caso de la ZMES de Europa Occidental, así como a las tendencias internacionales que procuran una revisión ordenada de las Directrices sobre ZMES. Aunado a ello, se señalan a manera de conclusiones, los derroteros que a futuro podría tomar esta asignatura tan - especialmente sensible-.

\section{LA LIBERTAD DE NAVEGACIÓN}

\section{Antecedentes}

El principio de la libertad de los mares, en el cual se encuadra el concepto normativo actual conocido como la libertad de navegación, es uno de los pilares de la evolución del derecho internacional. Su importancia es tal, que sin su efectiva aplicación sería imposible pensar en el comercio marítimo en el que se sustenta una parte importante de la economía mundial. ${ }^{4}$

Mientras que la Antigüedad Occidental se caracterizó por la imposición exclusiva del ejercicio de la navegación entre los pueblos conquistadores, como en su momento lo fueron los fenicios, los cartagineses o los helenos respecto al Mediterráneo, la era de la paz romana tuvo por primera vez en cuenta el criterio de juristas, entre ellos Ulpiano y Celso, quienes atribuían una connotación de uso común a los espacios marítimos. ${ }^{5}$

4 Véase el debate histórico sobre el principio de la libertad de los mares, consagrado hoy en la Convemar, artículo 87, en Grocio, Hugo, De la libertad de los mares, trad. de Blanco García y García Arias, Madrid, Civitas, 1979, pp. 53 y ss.; Fulton, Thomas, The Sovereignty of the Sea, Londres, 1911, pp. 17 y ss.; García, Luis, Historia del principio de la libertad de los mares, Santiago de Compostela, 1948, pp. 9 y ss.; Enríquez, David, "La libertad de navegación", Revista Jurídica Jalisciense, Guadalajara, enero-abril de 1997.

5 Véase D. 8.4.13 y D. 43.8.3. 
Desde luego, la interpretación que los gobernantes romanos daban a los criterios de sus jurisconsultos, no podía tener otra dimensión que la de garantizar el libre ejercicio de la navegación al interior del propio imperio romano, con lo cual, la vigencia del principio de la libertad de los mares entre distintas jurisdicciones, es en esta época, naturalmente cuestionable.

Con la caída del imperio romano y la consolidación de prósperas ciudades-Estado durante la Edad Media, creció la confrontación entre potencias marítimas, como Venecia o Génova, que buscaban reclamar derechos exclusivos sobre zonas marinas estratégicas para sus intereses comerciales. Conflictos similares emergieron no sólo en el Mar Mediterráneo sino también en los mares Báltico y del Norte; en donde Suecia, Dinamarca e Inglaterra buscaban tomar el control de las rutas internacionales.

Fue sin duda el inicio de la época de la colonización, la que llevó al punto más alto la discusión sobre la primacía en los mares. En ella, las pretensiones de las coronas aumentaban en proporciones desconocidas hasta el momento: España reclamaba derechos exclusivos sobre el Océano Pacífico y el Golfo de México, y Portugal sobre los océanos Índico y Atlántico Sur. ${ }^{6}$

\section{La batalla libresca}

Si bien la causa del estudio más famoso por la defensa de la libertad de los mares no fue necesariamente académica o altruista, no queda duda que elevó el debate de una simple discusión política y militar entre potencias marítimas a un verdadero debate jurídico entre pensadores, que a la postre influiría en la consolidación del derecho internacional del mar. Nos referimos a la obra de Hugo Grocio, conocida como Mare Liberum de $1605 .^{7}$

6 Las disputas por el control de los mares son, sin duda, cuna del derecho internacional. Si bien la jerarquía que éstas cobraron en la evolución del derecho internacional escapa al objeto de este trabajo, son recomendables entre otros muchos estudios: Basave, Agustín, Filosofía del derecho internacional, México, UNAM, IIJ, 1992, pp. 238 y ss.; Sorensen, Max, Manual de derecho internacional público, México, FCE, 1968, pp. 344 y ss.; Sepúlveda, César, Derecho internacional, México, Porrúa, 1991, pp. 463 y ss.

7 La obra, cuyo título completo es originalmente Mare liberum sive de jure quod Batavis competit ad Indicana comercia dissertatio es realmente uno de los capítulos del De iure praede commentarius, en el cual Grocio argumentaba a favor de la Compañía Holandesa de Indias 
En esencia, Mare Liberum fue una interesante argumentación escrita contra Portugal, publicada en oposición a España y utilizada en detrimento de Inglaterra por comerciantes marítimos holandeses, quienes lejos de defender los postulados filosóficos de la libertad de los mares - cuyo auténtico fundamento fue realmente labrado por los intelectuales españoles Francisco de Vitoria y Fernando Vázquez de Menchaca, medio siglo atrás - pretendían tener acceso a mejores tráficos y abundantes caladeros en los mares de sus vecinos.

Así, mediante la sistematización de los postulados de Vitoria y Vázquez de Menchaca, el edificio argumentativo del joven Grocio descansó en tres pilares, bien expuestos por Luis García con la redacción propia del texto original: ${ }^{8}$

En virtud del ius communicationis, los hispánicos no pueden prohibir a los holandeses el acceso a las Indias Orientales debido a: (i) no tener los lusitanos soberanía sobre la India, ni poder presentar a su favor títulos legítimos, que no pueden basarse en el ius inventionis, la donación pontificia o el ius belli; (ii) no tener dominio sobre el mar o sobre la navegación ni por descubrimiento u ocupación, ni por donación pontificia, ni por prescripción o costumbre adquisitiva; y (iii) no tener derecho a impedir el comercio, que es libre por derecho de gentes, ni por ocupación, ni por donación pontificia, ni por prescripción o usucapión, ni por motivos de equidad. Por consiguiente, los holandeses deben mantener el comercio con las Indias orientales, sea en paz, en tregua o en guerra, contra quien se oponga.

La famosa obra de Grocio dio lugar a uno de los debates más interesantes generados en el derecho internacional, la llamada "gran batalla libresca", una polémica intelectual - con dimensiones políticas e incluso bélicas - entre juristas de diversas nacionalidades, quienes defendían en uno y otro sentido la libertad o la exclusividad de los mares.

Orientales, quien a su vez buscaba convencer a sus accionistas menonitas de aceptar los beneficios económicos de la carraca portuguesa "Catalina", apresada en el litoral de la Península de Malaca en el sudeste asiático por el almirante Heemskerk en 1602. La obra fue escrita entre 1604 y 1605, cuando su autor contaba sólo con veinte años de edad. Véase el prólogo de Luis García en Grocio, Hugo, op. cit., nota 4, pp. 10 y ss. Véase también Brown, James, "La gènese du Traitè du Droit de la Guerre et de la Paix", Revue de Droit International et de Législation Comparée, Bruselas, 1925, t. VI, pp. 489 y ss.

8 Véase García, Luis, op. cit., nota 4, pp. 10 y ss. El autor hace una excelente relación de los autores, obras y sentidos argumentativos de la famosa polémica libresca protagonizada durante buena parte del siglo XVII. 
Si bien dentro de su contexto se redactaron documentos influyentes como los de William Welwood o Fray Serafín de Freitas, sería hasta 1618 en que el inglés John Seldein escribiría De dominio maris regio, publicado en 1635 bajo el título polémico de Mare clausum, en donde en oposición al Mare liberum de Grocio, argumentaría brillantemente desde una perspectiva histórica a favor de la política exclusivista inglesa sobre los mares. ${ }^{9}$

Como acertadamente explica Alejandro Sobarzo, la semilla echada por Grocio cayó en tierra fértil. A pesar de los vaivenes de la argumentación y la presión política entre las potencias marítimas, la balanza se inclinaría claramente hacia el principio de la libertad de los mares en oposición a la pretendida exclusividad de diversas potencias, en especial Inglaterra.

Así las cosas, otro holandés, Cornelio Van Bynkershoek, concluiría en 1702 con un siglo de discusiones mediante la publicación de su estudio, De dominio maris dissertatio, en el cual esbozaría la realidad en un principio: la potestad terrestre termina donde termina la fuerza de las armas. Con ello, la división entre el mar territorial y el alta mar tenía una sola medida efectiva: la de la fuerza de los cañones en batalla. ${ }^{10}$

\section{La Convención de las Naciones Unidas} sobre el Derecho del Mar

Finalmente, ya en el primer cuarto del siglo XIX, el equilibrio de potencias marítimas generado por la consolidación de grandes ejércitos en tierra hizo incuestionable el principio de la libertad de los mares. Sin embargo, faltarían todavía un siglo y medio más para quedar consagrado en el instrumento más importante y ampliamente sancionado en la historia del derecho del mar: la Convención de las Naciones Unidas sobre el Derecho del Mar de Montego Bay Jamaica de 1982 (Convemar), en donde

9 Idem. Véase también Fulton, Thomas, The Sovereingty of the Sea, Londres, Pitman, 1911, pp. 346 y ss.

10 A lo largo de las negociaciones por unificar las pretensiones sobre derechos exclusivos en la alta mar, se llegó por fin en la Convemar de 1982 al consenso que consiste en que todo Estado tiene derecho a establecer la anchura de su mar territorial hasta un límite que no exceda de 12 millas marinas medidas a partir de líneas de base determinadas de conformidad con la propia convención. Convemar, artículo 3. 
se establece de una vez y para siempre que ningún Estado podrá pretender legítimamente someter cualquier parte de la alta mar a su soberanía. ${ }^{11}$

En efecto, harían falta tres conferencias de las Naciones Unidas sobre Derecho del Mar para dotar de validez formal plena a la libertad de navegación. Con un trabajo iniciado en 1949 por la Comisión de Derecho Internacional de las Naciones Unidas y desarrollado a lo largo de más de tres décadas hasta la Convención de Jamaica, la libertad de navegación debe entenderse como uno de los seis derechos reconocidos por la Convemar a sus Estados miembros.

Así, de acuerdo con el artículo 87 de dicho instrumento internacional, libertad de navegación, libertad de sobrevuelo, libertad de tendido de cables y tuberías submarinos, libertad de construcción de islas artificiales y otras instalaciones permitidas por el derecho internacional, libertad de pesca y libertad de investigación científica constituyen la carta de derechos reconocidos a todos los Estados, sean éstos ribereños o sin litoral.

La libertad en el ejercicio de los derechos mencionados en la alta mar está sujeta a los límites tanto de la propia Convemar, como de otras normas de derecho internacional; y debe siempre tener en cuenta los intereses de otros Estados en su ejercicio legítimo.

\section{LAS ZONAS MARINAS ESPECIALMENTE SENSIBLES}

\section{La Convemar y otras normas internacionales}

Además de los deberes generales impuestos a cada Estado contratante de la Convemar como correlativos a sus derechos sobre la alta mar y las demás zonas marinas, todo Estado contratante tiene la obligación de proteger y preservar el medio marino.

Dentro de las medidas para prevenir, reducir y controlar la contaminación del medio marino, debemos subrayar por ser de interés del tema de las ZMES, la obligación de que, entre las medidas que se tomen de acuerdo a la Convemar, figuren las necesarias para proteger y preservar los ecosistemas raros o vulnerables, así como el hábitat de las especies y otras formas de vida marina diezmadas, amenazadas o en peligro. ${ }^{12}$ 
Antes de profundizar en las normas ambientales de la Convemar, conviene hacer una breve reflexión sobre el entramado de las normas internacionales marítimas especiales, tendentes directa o indirectamente a la protección del medio marino. Como puede apreciarse de los diversos instrumentos obligatorios y de naturaleza no vinculante de la OMI, el objeto de todos ellos - y en particular de SOLAS y MARPOL - es regular la integridad estructural de los buques, su equipo, su tripulación y en general, su funcionamiento. Las normas inscritas en dichos convenios promueven la seguridad en el transporte marítimo y en consecuencia, protegen a los ecosistemas marinos de la contaminación.

En este sentido, puede sintetizarse el trabajo de la OMI en materia de protección del medio marino - directo e indirecto - en cuatro categorías de medidas: (i) el Convenio SOLAS, diseñado para la seguridad del sector naviero y su efecto indirecto en la protección ambiental; (ii) el Convenio MARPOL en el que se dispone un nivel básico de protección ecológica tanto de las descargas operacionales como de las accidentales; (iii) las medidas de organización del tráfico marítimo, aprobadas para incrementar la seguridad en la navegación y que a su vez coadyuvan en la protección de los ecosistemas marinos; y (iv) la designación de zonas especiales —ZME - en las cuales las prescripciones de descarga son más estrictas de acuerdo a lo detallado en los anexos I, II y V de MARPOL; y de ZMES en los casos que en este estudio se comentan.

En adición a las labores de la OMI a través de instrumentos como MARPOL o SOLAS, existen otras fuentes de derecho internacional - además de la propia Convemar- a las ZMES. En efecto, la Convención de las Naciones Unidas sobre la Diversidad Biológica constituye un instrumento internacional mediante el cual, entre otros aspectos, se establece un marco propicio para designar zonas como especialmente protegidas en lugares donde se presentan elevadas concentraciones de recursos biológicos vulnerables. ${ }^{13}$

En este contexto de fuentes de derecho internacional, la Convemar reconoce ciertas categorías de zonas marinas que requieren mayores niveles de protección ambiental: estas son precisamente las ZMES. Una vez subrayada la importancia de las ZMES y la necesidad de aplicar en ellas normas ambientales más estrictas que a otros espacios marinos, la

13 Véase en especial, el artículo 8 de dicha convención, en la cual se regula la designación de zonas protegidas y confrontar sus disposiciones con las directrices mencionadas en este trabajo. 
Convemar establece en una serie de reglas el criterio de legitimación y el procedimiento para la determinación de ZMES y sus respectivas medidas de protección ambiental: 14

a) De acuerdo a la Convemar, cuando las reglas y estándares internacionales para prevenir, reducir y controlar la contaminación del medio marino causado por buques sean inadecuados para hacer frente a circunstancias especiales, y los Estados ribereños tengan motivos razonables para creer que un área particular y claramente definida de sus respectivas zonas económicas exclusivas requiere la adopción de medidas obligatorias especiales para prevenir la contaminación por buques, por reconocidas razones técnicas relacionadas por sus condiciones oceanográficas y ecológicas, así como por su utilización o la protección de sus recursos y el carácter particular de su tráfico, los Estados ribereños, tras celebrar consultas apropiadas a través de la organización — misma que se entiende como la OMI - con cualquier otro Estado interesado, podrá dirigir una comunicación a dicha organización internacional, presentando pruebas científicas y técnicas en su apoyo.

b) Dentro de los doce meses siguientes al recibo de tal comunicación - sigue la Convemar - la organización determinará si las condiciones en esa área corresponden a los requisitos enunciados. Si la organización así lo determina, los Estados ribereños podrán dictar para esa área leyes y reglamentos destinados a prevenir, reducir y controlar la contaminación causada por buques, aplicando las reglas y estándares o prácticas de navegación internacionales que, por conducto de la organización, se hayan hecho aplicables a las áreas especiales. Esas leyes y reglamentos no entrarán en vigor para los buques extranjeros hasta quince meses después de haberse presentado la comunicación a la organización. Los Estados ribereños deben publicar los límites de tal área particular y claramente definida.

c) Al presentar su solicitud, los Estados ribereños deben manifestar si tienen intención en dictar leyes y reglamentos adicionales sobre la ZMES que vaya a ser determinada dentro del trámite. Si bien tales leyes y reglamentos pueden referirse a descargas o prácticas de la navegación, no pueden obligar a los buques extranjeros a cumplir estándares de diseño, construcción, dotación y equipo distintos de las reglas internacionalmente aceptadas. Las nuevas leyes y reglamentos del Estado ribereño 
pueden ser aplicadas a los buques extranjeros quince meses después de haberse presentado la solicitud a la organización, a condición de que ésta dé su conformidad dentro de los doce meses siguientes a su presentación.

d) Las reglas y estándares internacionales a los que antes se ha aludido deben comprender, en particular, los relativos a la pronta notificación a los Estados ribereños cuyo litoral o intereses conexos puedan resultar afectados por incidentes, incluidos accidentes marítimos que ocasionen o puedan ocasionar descargas.

\section{La OMI y las directrices internacionales}

\section{A. La gestación de las directrices}

Además de las obligaciones internacionales de Convemar antes descritas, como acertadamente señala Paul Nelson, las ZMES tienen sus orígenes técnicos en la Resolución 9 de la Conferencia Internacional sobre Seguridad de Buque-Tanques y la Prevención de la Contaminación de febrero de 1978, en la cual se incluyeron una serie de medidas sobre el diseño y operación de ese tipo de embarcaciones, mismas que quedaron a su vez incorporadas en los protocolos del mismo año a los convenios internacionales para prevenir la contaminación por los buques (MARPOL) y para la seguridad de la vida humana en el mar (SOLAS). ${ }^{15}$

La Resolución 9 reconocía como ZMES a las zonas marinas, respecto de las cuales se tuviera una especial necesidad de protección contra la contaminación marina proveniente de buques y vertimientos, teniendo en cuenta la característica renovable de sus recursos o bien la importancia de la misma para propósitos científicos. A partir de la referencia de 1978 sobre las ZMES, el Comité de Protección del Medio Marino (CPMM o más conocido por sus siglas en inglés MEPC) de la OMI se dio a la tarea de generar una serie de criterios objetivos para la identificación y determinación de ZMES. El trabajo del MEPC continuó por espacio de varios años, hasta que se llegó en 1991 a la aprobación por parte de la Asamblea de la OMI de la Resolución A.720(17) en la cual se contenían las primeras Directrices para la Identificación y Designación de ZMES.

15 Nelson, Paul, "Protecting areas that are vulnerable to damage by maritime activities: the reality of particularly sensitive sea areas", Maritime Studies, julio-agosto de 2003, pp. 20 y ss. 
A pesar de estar listas para ser usadas por los países miembros en sus solicitudes de ZMES, la Tercera Sesión Internacional de Expertos Jurídicos en ZMES, llevada a cabo en Países Bajos en 1994, expresó su preocupación, pues desde su aprobación en 1991 solamente una solicitud - la de la Gran Barrera de Coral australiana - se había presentado, con lo cual se decidió revisar las directrices. Así las cosas, en 1997 el MEPC constituyó un grupo por correspondencia en donde se evaluara la necesidad de revisar las directrices. La labor del grupo tuvo como resultado la aprobación de las directrices vigentes aprobadas de acuerdo a la Resolución A.927(22) de la Asamblea de la OMI en noviembre de 2001.

El documento, titulado Directrices para la Designación de Zonas Especiales en virtud de MARPOL 73/78 y Directrices para la Determinación y Designación de Zonas Marinas Especialmente Sensibles, ${ }^{16}$ es el instrumento vigente en el que todo Estado miembro debe basarse para solicitar una ZMES, y en el que los órganos competentes de la OMI se fundamentan para su determinación. Ahora bien, en marzo de 2003, el MEPC aprobó una circular en donde se contiene un documento pro-forma que pretende servir de modelo para los solicitantes de ZMES. ${ }^{17}$

\section{B. Elementos distintivos entre las ZME y las ZMES}

Con el fin de comprender mejor el contenido de las Directrices, conviene establecer primero la diferencia entre las ZME y las ZMES. Las ZME son definidas en MARPOL como cualquier extensión de mar en la que, por razones técnicas reconocidas en relación con sus condiciones oceanográficas y ecológicas, y el carácter particular de su tráfico marítimo, se hace necesario adoptar procedimientos especiales obligatorios para prevenir la contaminación del mar por hidrocarburos, sustancias nocivas líquidas o basuras, según sea el caso. ${ }^{18}$

Por su parte, las ZMES - cuyo fundamento principal se encuentra en la Convemar - son aquellas que deben ser objeto de protección especial, de acuerdo con las medidas que adopte la OMI, en atención a su importancia por motivos ecológicos, socioeconómicos o científicos reconoci-

16 En adelante, "las Directrices". De acuerdo con la Resolución A. 927(22) las resoluciones A.720(17) y A.885(21) fueron revocadas.

17 MEPC/Circ.398. En adelante "el Documento Modelo".

18 MARPOL 73/78, anexos I, II y V. 
dos, y a que su medio ambiente puede sufrir daños como consecuencia de las actividades marítimas. ${ }^{19}$

Por las características y propósitos distintos entre las ZME y las ZMES, el CPMM decidió derivar la Resolución A22/Res.927 de la asamblea en dos anexos: uno para las ZME y otro para las ZMES. Como puede apreciarse, una ZME no goza de la connotación ambiental más estricta como sí ocurre en cambio con una ZMES. Es decir, una ZMES - y no una $\mathrm{ZME}$ - se justifica en la medida que es un ecosistema raro o vulnerable, así como el hábitat de las especies y otras formas de vida marina diezmadas, amenazadas o en peligro. ${ }^{20}$

Ahora bien, de acuerdo a uno de los criterios establecidos por las Directrices, en muchos casos se podrá determinar que una zona marina que se encuentra dentro de una zona especial, es especialmente sensible, y viceversa. Cabe señalar que los criterios para determinar zonas marinas especialmente sensibles y los criterios para designar zonas especiales no se excluyen mutuamente.

\section{Consideraciones generales en la designación de ZMES}

Las Directrices tienen tres finalidades básicas. Primero, buscan proporcionar orientación a los Estados miembros de la OMI en cuanto a la formulación y presentación de solicitudes para la designación de ZMES. En segundo lugar, pretender garantizar que en ese proceso se consideren escrupulosamente todos los intereses, tanto los del Estado ribereño, como los del Estado de abanderamiento, los colectivos interesados en el medio ambiente y el sector del transporte marítimo; teniendo en cuenta la información científica, técnica, económica y medioambiental pertinente sobre la zona expuesta a riesgos por las actividades marítimas internacionales, así como las medidas de protección para reducir al mínimo dichos riesgos. Finalmente, la Directrices tienen la intención de prever los elementos técnicos necesarios para que la OMI evalúe las solicitudes.

Ahora bien, la determinación de toda ZMES y la adopción de las correspondientes medidas de protección exigen examinar tres elementos: $i$ ) las condiciones medioambientales concretas de la zona que debe determinarse; ii) la vulnerabilidad de dicha zona a los daños causados por las 
actividades marítimas internacionales; y iii) la competencia de la OMI para disponer las correspondientes medidas de protección frente a los riesgos que presentan las actividades marítimas. ${ }^{21}$

\section{Las actividades maritimas internacionales y el medio marino}

Para conocer la manera en la que inciden las actividades marítimas en una determinada zona, es necesario primero conocer las categorías de riesgos que ésta podría sufrir. En general, las actividades marítimas ordinarias son susceptibles de producir tres especies de peligros para el ambiente: i) descargas resultantes de las operaciones; ii) contaminación accidental o intencionada; y iii) daños físicos a los hábitats u organismos marinos.

Como se explica en las Directrices, durante las operaciones normales y en casos de accidente, los buques pueden descargar una gran variedad de sustancias contaminantes, bien directamente en el medio marino, bien indirectamente a través de la atmósfera. Esos contaminantes pueden ser hidrocarburos y mezclas oleosas, sustancias nocivas líquidas, aguas sucias, basuras, sustancias nocivas sólidas, pinturas antiincrustantes, organismos foráneos, e incluso ruido.

Muchos de estos elementos pueden afectar desfavorablemente al medio marino y a los recursos vivos del mar. Asimismo, los contaminantes pueden dañar el medio ambiente en caso de accidente marítimo. Además, los buques pueden ocasionar daños a los organismos marinos y a sus hábitats por impacto físico. Las varadas pueden asfixiar los hábitats, y se han dado incluso casos de colisiones entre buques y grandes cetáceos como las ballenas.

\section{E. Criterios ecológicos, socioeconómicos y científicos} para la determinación de una ZMES

Para ser clasificada por la OMI como una ZMES, la zona de que se trate debe, por una parte, satisfacer al menos uno de los criterios ecológicos, socioeconómicos o científicos que en esta sección se señalan; y por la otra, ser vulnerable a las actividades marítimas internacionales a partir 
de los factores que se indicarán en la sección subsiguiente. Así pues, se expone primero cada uno de los criterios a considerar, para posteriormente hacer referencia a los factores de vulnerabilidad derivados de las actividades marítimas.

\section{a. Criterios ecológicos}

Singularidad o rareza. Los ecosistemas pueden ser únicos o raros. Una zona o un ecosistema son únicos cuando no hay más que uno en su género. Ejemplo de ello son los hábitats de especies raras, amenazadas o en peligro de extinción que se dan en una sola zona. Una zona o un ecosistema son raros cuando sólo se dan en unos pocos lugares o cuando todos los de su clase están en franca regresión. Los ecosistemas pueden rebasar las fronteras nacionales y revestir importancia regional o internacional. Los criaderos o determinadas zonas de alimentación también pueden ser únicos o raros.

Hábitats críticos. Una zona marina puede constituir un hábitat crítico para una población de peces o especies marinas raras o en peligro, o bien, tener una importancia decisiva para mantener grandes ecosistemas marinos.

Dependencia. Los fenómenos ecológicos de tales zonas dependen en gran medida de la estructura biótica de los sistemas. ${ }^{22}$ A menudo, esos ecosistemas de estructura biótica presentan una gran diversidad que depende de los organismos constituyentes. La dependencia abarca también zonas que constituyen las rutas migratorias de peces, reptiles, aves y mamíferos marinos.

Carácter representativo. Las zonas son extremadamente representativas de los fenómenos ecológicos, de los tipos de comunidad o de hábitat o de otras características naturales. La representatividad corresponde al grado en que la zona representa un tipo de hábitat, un fenómeno ecológico, una comunidad biológica, una característica fisiográfica u otra característica natural.

Diversidad. Las zonas cuentan con gran variedad de especies o diversidad genética, o incluyen una multiplicidad de ecosistemas, hábitats y comunidades. No obstante, este criterio puede no ser aplicable a ciertos

22 Por ejemplo, arrecifes de coral, bosques de algas pardas, manglares y lechos de zosteras y algas marinas. 
ecosistemas más simples, por ejemplo a algunas comunidades pioneras o en equilibrio ecológico, ni a zonas sometidas a fuerzas destructivas, tales como los litorales expuestos a la acción violenta de las olas.

Productividad. La zona presenta una gran productividad biológica natural. Esa producción es el resultado de procesos biológicos y físicos que culminan en un aumento neto de biomasa en zonas de gran productividad natural, tales como frentes oceánicos, zonas de corrientes ascendentes y algunos giros oceánicos.

Zonas de desove o reproducción. La zona puede ser un lugar de desove o de reproducción de especies marinas que pasen el resto de su ciclo vital en otras zonas, o una ruta migratoria de aves y mamíferos marinos.

Carácter natural. La zona tiene un carácter altamente natural por haber escapado a las perturbaciones y la degradación causadas por el hombre.

Integridad. La zona constituye una unidad biológicamente funcional, es decir, una entidad ecológica autónoma viable. Cuanto más autosuficiente sea la zona desde el punto de vista ecológico, mayor será la probabilidad de que su valor pueda protegerse eficazmente.

Vulnerabilidad. La zona es muy susceptible a la degradación ocasionada por los fenómenos naturales o las actividades humanas. Las comunidades bióticas de los hábitats costeros pueden presentar una baja tolerancia a los cambios en las condiciones ambientales, o existir cerca de su umbral de tolerancia. ${ }^{23}$ También pueden verse expuestas a perturbaciones naturales, como tormentas o emersión prolongada, que determinen los límites de su desarrollo. Otras condiciones desfavorables ${ }^{24}$ pueden determinar la recuperación total o parcial de la zona de los efectos de las perturbaciones naturales, o su destrucción.

Más aún, algunos factores oceanográficos y meteorológicos podrían hacer vulnerable una zona o aumentar su vulnerabilidad; por ejemplo, causando la concentración o retención de sustancias perjudiciales en las aguas o en los sedimentos, o haciendo que quede expuesta a las sustancias perjudiciales. Dichos factores incluyen tipos particulares de circulación de las aguas, tales como zonas de convergencia, frentes y giros oceánicos, o tiempo de presencia prolongado resultante de las bajas tasas

23 Determinado ello por la temperatura, salinidad, turbiedad o profundidad de las aguas.

24 Tales como la contaminación de origen doméstico o industrial, la reducción excesiva de la salinidad y el aumento de la turbiedad provocada por una mala gestión de la cuenca. 
de dispersión, una estratificación por densidad permanente o estacional que puede conducir a un empobrecimiento del oxígeno de la capa del fono, así como condiciones desfavorables de hielo o de viento. Una zona cuyo medio ambiente ya está sometido a tensiones producidas por actividades humanas o por fenómenos naturale ${ }^{25}$ puede necesitar protección especial contra tensiones adicionales, incluidas las derivadas de las actividades marítimas internacionales.

Importancia biogeográfica. La zona tiene características biogeográficas poco comunes o es representativa de un tipo o tipos biogeográficos, o presenta características geológicas únicas, o poco comunes.

\section{b. Criterios socioeconómicos y culturales}

Beneficios económicos. La zona reviste especial importancia para el aprovechamiento de los recursos marinos vivos.

Recreo. La zona ofrece un interés particular para las actividades recreativas y el turismo.

Dependencia humana. La zona es particularmente importante para los modos de subsistencia y/o las necesidades culturales tradicionales de la población local.

c. Criterios científicos y pedagógicos

Investigación. La zona reviste gran interés científico

Estudios de referencia y de vigilancia. La zona reúne las condiciones de referencia apropiadas en lo que respecta a la biota o a las características medioambientales.

Educación. La zona ofrece la oportunidad de demostrar determinados fenómenos naturales. 
F. Factores de vulnerabilidad derivados de las actividades maritimas

a. Factores del tráfico marítimo

Factores operacionales. Tipos de actividades marítimas en la zona propuesta que puedan acrecentar el riesgo para la seguridad de la navegación. ${ }^{26}$

Tipos de buques. Tipos de buques que pasan por la zona o por una zona adyacente a la propuesta. ${ }^{27}$

Características del tráfico. El volumen o concentración de tráfico, la interacción entre buques, la distancia a la costa $u$ otros peligros para la navegación que aumentan el riesgo de abordaje o varada.

Sustancias perjudiciales transportadas. Tipo y cantidad de sustancias a bordo, ya se trate de carga, combustible o provisiones, que serían perjudiciales si se descargasen en el mar.

$b$. Factores naturales de la zona

Hidrográficos. Profundidad del agua, topografía del fondo marino y del litoral, ausencia de fondeaderos próximos y seguros, y otros factores que requieren la adopción de mayores medidas de precaución en la navegación.

Meteorológicos. Tiempo preponderante, fuerza y dirección del viento, visibilidad atmosférica y otros factores que aumentan el riesgo de abordaje y varada, así como el riesgo de que la zona sufra daños en caso de producirse un derrame.

Oceanográficos. Corrientes de marea, corrientes oceánicas, hielos y otros factores que aumentan el riesgo de abordaje y varada, así como el riesgo de que la zona sufra daños en caso de producirse un derrame.

\section{G. Medidas de protección correspondientes}

Como se ha comentado, la determinación de una ZMES por sí misma no tiene sentido si no va acompañada de una serie de medidas de protec-

26 Por ejemplo, pequeñas embarcaciones pesqueras, pequeñas embarcaciones de recreo, plataformas petroleras y gaseras, etcétera.

27 Por ejemplo, naves de gran velocidad, buques tanque de grandes dimensiones o graneleros con poca profundidad de agua bajo la quilla. 
ción que tengan en cuenta los criterios ecológicos, socioeconómicos y científicos, además de los factores de vulnerabilidad propios de la ZMES en cuestión. Por supuesto, las medidas de protección están limitadas al ámbito de facultades de la OMI y se concretan en el siguiente catálogo:

a) Designar el lugar de que se trate como zona especial en virtud de los anexos I, II o V del Convenio MARPOL, o zona de control de las emisiones de Sox en virtud del anexo VI del mismo convenio; o bien aplicar restricciones especiales a las descargas de los buques que operen en dicha zona. ${ }^{28}$

b) Adoptar sistemas de notificación para buques y de organización del tráfico marítimo, en virtud del Convenio SOLAS, y de conformidad con las disposiciones generales sobre organización del tráfico marítimo y las Directrices y criterios relativos a los sistemas de notificación para buques, en las ZMES y sus inmediaciones. ${ }^{29}$

c) Elaborar y adoptar otras medidas destinadas a proteger determinadas zonas marinas contra daños ambientales ocasionados por los buques, tales como sistemas de practicaje obligatorio o sistemas de regulación del tráfico marítimo. En realidad, esta categoría de medidas de protección es lo suficientemente amplia para que de acuerdo al caso concreto, el Estado miembro solicitante diseñe la medida específica necesaria para la protección de la zona.

Además de las medidas de protección autorizadas por OMI a partir de la solicitud, con el propósito de fortalecer la jerarquía de la ZMES como tal, es conveniente examinar la posibilidad de incluirla en la Lista del Patrimonio Mundial; declararla Reserva de la Biosfera; o bien incluirla en una lista de zonas de importancia internacional, regional o nacional. Es asimismo necesario que el solicitante señale si la zona ya es objeto de medidas o acuerdos de conservación internacionales, regionales o nacionales. ${ }^{30}$

28 Debe destacarse que los procedimientos y criterios para la designación de zonas de control de las emisiones de Sox figuran en el anexo VI de MARPOL.

29 En esta medida de protección, hay en realidad tres alternativas a ser reguladas: (i) evitar la zona totalmente; (ii) establecer medidas especiales de organización del tráfico marítimo; o bien, (iii) establecer medidas para notificación para buques.

30 En el caso mexicano será necesario indicar si la zona marina propuesta a OMI está en el Sistema Nacional de Áreas Protegidas, y en su caso, cuál es el tratamiento técnico y jurídico de la misma. Si bien la OMI no está obligada a reflejar las medidas de protección que autorice las normas propias del país solicitante — en este caso México - naturalmente tomará en cuenta lo ya regulado y en práctica por el gobierno solicitante. 
Si las circunstancias de la ZMES en cuestión lo ameritan, la propuesta puede también incluir una zona de separación; esto es, un área contigua al lugar específico o zona central que se desea proteger del tráfico marítimo. Para ello, es preciso justificar la necesidad de la zona de separación y en qué medida ésta aporta a la protección efectiva de la zona central.

\section{H. Procedimiento para la designación de ZMES y sus medidas de protección}

Cuando se presenten solicitudes para la designación de ZMES que no contengan propuestas para la adopción de medidas de protección, el Estado miembro solicitante debe informar sobre el tipo de medidas que esté considerando. Debe tenerse en cuenta que en un plazo máximo de dos años, a partir de la aprobación en principio de una ZMES por el MEPC, deberá presentarse al menos una propuesta de medida de protección correspondiente. ${ }^{31}$

La solicitud debe contener un resumen de los objetivos de la propuesta, de la situación de la zona, la necesidad de protegerla y las medidas de protección correspondiente que se piden. El resumen debe explicar los argumentos por los que las medidas propuestas de protección correspondiente constituyen el método preferible para proteger la zona cuya determinación como ZMES se solicita.

En esencia, las solicitudes de designación deben tener en cuenta todas las consideraciones y criterios que figuran en las Directrices e incluir la información que demuestre cada uno de ellas; y en su caso, el tratamiento y medidas que ya el Estado miembro lleve a cabo para la protección de la zona marina que se pretende elevar a categoría de ZMES. De igual forma, es necesario señalar las normas nacionales en materia de sanciones por infracción, que el Estado miembro establecerá en su legislación respecto a las embarcaciones que incumplan las medidas de protección que vayan a ser autorizadas por la OMI.

Las solicitudes deben segmentarse en dos partes: la primera debe estar dedicada a la descripción, importancia y vulnerabilidad de la zona; la

31 Existen casos en que la OMI, desde antes de la solicitud de la ZMES por el Estado miembro, ha adoptado ciertas medidas de protección para toda esa zona o bien para una parte de ella, o incluso un conjunto de zonas. En esos casos, es necesario que el solicitante indique en qué forma las medidas ya adoptadas contribuyen a la protección de la ZMES en cuestión. 
segunda debe explicitar las medidas de protección correspondientes y destacar la competencia de la OMI para adoptarlas de acuerdo a los convenios internacionales aplicables. En la evaluación caso por caso de las solicitudes, la OMI está obligada a tener en cuenta los diversos elementos de las Directrices, y en particular si se agotan los siguientes tres criterios:

a) El conjunto de medidas de protección disponibles, y determinará si las medidas de protección correspondientes propuestas son apropiadas para hacer frente con eficacia al riesgo evaluado de que determinadas actividades marítimas internacionales produzcan daños en la zona propuesta.

b) Si tales medidas aumentarían la posibilidad de que dichas actividades marítimas internacionales tengan efectos negativos importantes en el medio ambiente fuera de la ZMES propuesta.

c) Si la extensión de la zona se limita a la necesaria para satisfacer las necesidades identificadas.

Una vez presentada la solicitud al MEPC, de acuerdo con las Directrices, la OMI, de conformidad con los criterios de evaluación señalados en el párrafo anterior, debe agotar el siguiente procedimiento de análisis y resolución en seis etapas específicas que brevemente se describen a continuación:

a) El MEPC es el primer responsable de estudiar las solicitudes de determinación de ZMES, por lo que todas las solicitudes se presentarán - como se ha ya mencionado- en primer lugar a dicho comité.

b) El MEPC examinará inicialmente la solicitud para establecer si se ajusta a las disposiciones de las Directrices. En caso afirmativo, el comité podrá aprobar en principio la determinación de la zona propuesta como ZMES, y remitirá la solicitud, junto con sus correspondientes medidas de protección, al subcomité o comité competente encargado de las correspondientes medidas de protección concretas que se proponen para la zona; quien a su vez podrá pedir asesoramiento al MEPC acerca de cuestiones relativas a la solicitud. En cualquier caso, el MEPC está impedido para tomar una decisión definitiva en cuanto a su determinación, hasta que el subcomité o comité pertinente haya estudiado las medidas de protección correspondientes; ${ }^{32}$

32 Es curioso que las Directrices señalan que el comité competente podría ser el propio MEPC, por lo cual se remitiría el caso a sí mismo. Nada impide, sin embargo, que de hacer esto, pueda el 
c) Por lo que respecta a las medidas que exigen la aprobación del Comité de Seguridad Marítima (CSM o más conocido por sus siglas en inglés MSC), el subcomité presentará al MSC la recomendación de que apruebe dichas medidas; o bien, en caso de rechazarlas, el subcomité informará de ello tanto al MEPC como al MSC, exponiendo las razones de su decisión. Por su parte, el MSC estudiará las recomendaciones que se le hagan y, si decide que se adopten las medidas, lo notificará al MEPC.

d) Si se presenta una solicitud en la que no se propongan las medidas de protección correspondientes, el MEPC podrá aprobar en principio la determinación de la zona como ZMES, a reserva de que en un plazo máximo de dos años a partir de la aprobación se presente al menos una propuesta de medida de protección correspondiente y seguidamente se apruebe como mínimo, una de dichas medidas de protección. ${ }^{33}$

e) Si la solicitud se rechaza, el MEPC deberá informar al Estado miembro proponente, y le presentará una exposición de los hechos que han motivado su decisión.

f) Una vez que el comité o subcomité competente haya aprobado las medidas de protección correspondientes, el MEPC podrá designar la zona - definitivamente- como ZMES.

En todo caso, de acuerdo a las propias Directrices, la OMI debe servir de foro para la revisión y nueva evaluación de toda medida de protección adoptada, según proceda, teniendo en cuenta los comentarios, informes y observaciones pertinentes sobre las medidas. Los gobiernos cuyos buques efectúan operaciones en la ZMES tienen el derecho de poner en conocimiento de la OMI las inquietudes que tengan respecto a las medidas de protección, de modo que se puedan hacer las modificaciones pertinentes a éstas. ${ }^{34}$

Finalmente, al evaluar cada caso, la OMI debe tener en cuenta los recursos técnicos y financieros de que disponen los Estados miembro en desarrollo, o aquellos cuyas economías están en periodo de transición de sistema. Esta norma es un reconocimiento al encarecimiento que un cam-

MEPC formar un grupo de trabajo que lo asesore sobre la solicitud de determinación y las medidas de protección relacionadas.

33 Lo anterior, salvo en el caso en que no se propongan medidas de protección, porque la OMI ya las hubiere adoptado antes.

34 Desde luego, el solicitante de la determinación de ZMES están también legitimados para poner en conocimiento de la OMI cualesquiera incertidumbres o propuestas de medidas suplementarias o modificaciones de dichas medidas de protección correspondientes. 
bio de ruta puede representar para las embarcaciones que tengan tráficos por o cercanos a la ZMES determinada.

\section{Implantación de las ZMES y medidas de protección correspondientes}

Toda vez que la solicitud de determinación de una ZMES y sus respectivas medidas de protección son de la mayor prioridad dentro de la política ambiental para cualquier país, tras la autorización de la OMI, luego de agotado el procedimiento, ésta se encuentra obligada a garantizar que la fecha de implantación efectiva sea lo más temprana posible, de conformidad con las normas aplicables.

Una vez aprobada la designación de una ZMES se deben indicar en todas las cartas náuticas todas las medidas de protección correspondientes, utilizando los símbolos y métodos de la Organización Hidrográfica Internacional (por sus siglas $\mathrm{OHI}$ ). Si bien los Estados miembro proponentes también pueden consignar en las cartas las ZMES designadas con los símbolos nacionales pertinentes, si la OHI adopta un símbolo internacional, aquél está obligado a señalar la ZMES en cuestión, utilizando dicho símbolo y los métodos recomendados por la OHI.

Los Estados miembro están obligados a adoptar todas las medidas necesarias para garantizar que las embarcaciones que enarbolan su pabellón cumplan las medidas de protección correspondientes adoptadas para proteger la ZMES designada. En este sentido, los Estados miembro que reciban información sobre una presunta infracción de una medida de protección por parte de un buque que enarbole su pabellón, debe facilitar al gobierno que haya notificado la infracción, los pormenores de toda medida adoptada al respecto.

\section{J. Las Directrices y el derecho del mar}

Las Directrices expresan constantemente el respeto que se debe tener en la designación de la ZMES y sus respectivas medidas de protección con el derecho internacional del mar, recogido en la Convemar. En efecto, como se ha recordado al inicio de este trabajo, el reconocimiento de la libertad de navegación ha sido objeto de un largo camino, para ser lo que es hoy, por lo que todos los instrumentos internacionales que de alguna forma limitan tal libertad, procuran destacar el respeto al derecho del mar. 
Las ZMES y, sobre todo, las medidas de protección significan auténticas limitaciones a la libertad de navegación en pro de un bien jurídico superior bajo tutela del derecho: la protección del medio marino. Esta protección, como se ha también advertido, guarda perfecta armonía con la Convemar pues la libertad de navegación no puede ser entendida como un derecho irrestricto de los Estados o sus nacionales, sino en la medida que sirvan a la conservación como un valor superior de la comunidad internacional.

Así pues, una determinación de ZMES y sus respectivas medidas de protección que no cumplan con los criterios establecidos en las Directrices, o bien que cumpliéndolos formalmente pretendan ir más allá de las propias Directrices, representaría un auténtico abuso del derecho. El delicado equilibrio alcanzado entre el legítimo derecho de los Estados en solicitar la determinación de ZMES y la libertad de navegación de los buques de todos los demás Estados hace que abusos potenciales en las solicitudes o en los procedimientos pongan en entredicho la vigencia efectiva del derecho del mar. ${ }^{35}$

Así pues, la OMI y los países que la integran deben permanecer siempre vigilantes de que las solicitudes de ZMES sean válidas y razonables, de modo que la armonía aludida entre la regulación para la protección del ambiente marino y la libertad de navegación coexistan sin abusos, por parte de países o regiones que cuentan con los medios económicos y técnicos necesarios para hacer pasar como auténticas ZMES a zonas marinas que, si bien sean relevantes, no reúnan las características suficientes para ser válidamente reconocidas como tales por la comunidad internacional. De igual forma es necesario velar para que la falta de recursos económicos o técnicos de países en desarrollo o en transición no sea un factor limitante para que zonas marinas que realmente merezcan ser reconocidas como ZMES, terminen siéndolo.

Hasta aquí se ha repasado tanto la evolución del derecho del mar, como la regulación en materia de ZMES y sus respectivas medidas de protección. Se ha advertido la necesidad de guardar el equilibrio entre protección del ambiente y libertad de navegación. Falta ahora analizar, a la luz de casos prácticos de actualidad - particularmente el relativo a la ZMES de Europa Occidental- si existen o no razones suficientes para temer que la vigencia del vetusto principio de la libertad de los ma- 
res se encuentra en franca decadencia por la autorización de ZMES de controvertida validez.

\section{EL CASO DE LAS ZMES DE EUROPA OCCIDENTAL}

\section{La propuesta: aspectos generales}

En el ámbito de la 49a. sesión del MEPC en abril de 2003, diversos países miembros de la Unión Europea - Bélgica, España, Francia, Irlanda, Portugal y Reino Unido - presentaron una propuesta para determinar una ZMES en la Europa Occidental que iría desde las Islas Shetland, al Norte, hasta el Cabo de San Vicente, al Sur, así como el Canal de la Mancha y sus accesos. ${ }^{36}$

Entre la justificación general que destacan los solicitantes, se encuentran los dramáticos accidentes marítimos que han tenido lugar a lo largo de los últimos años en aguas europeas, ${ }^{37}$ y en cómo éstos han puesto de manifiesto las deficiencias de la normatividad internacional, tanto en lo que se refiere a la seguridad marítima, como a la protección del medio marino y el litoral contra la contaminación accidental.

La propuesta de la ZMES de Europa Occidental debe entenderse como un paquete de medidas estratégicas orquestadas por la Comisión Europea, luego del accidente del Prestige en el 2003. Dichas medidas contemplan principalmente: a) la retirada acelerada de los buques tanque de casco sencillo de las aguas europeas; b) la creación de un fondo suplementario de indemnización para las víctimas de derrames de hidrocarburos, en el ámbito del Fondo Internacional de Indemnización de Daños Debidos a la Contaminación por Hidrocarburos -FIDAC_- y c) la determinación de una ZMES de la Europa Occidental con estrictas medidas de protección.

36 La ZMES de la Europa Occidental incluye determinadas partes de la zona especial conocida como "Aguas del Noroeste de Europa", tal como se definen en la Regla 10 (1) (h) del anexo I del Convenio MARPOL; determinadas partes de la zona de control de contaminación del Reino Unido; determinadas partes de la zona de respuesta a la contaminación de Irlanda; y determinadas partes de las zonas económicas exclusivas de Bélgica, Francia, España y Portugal. La presentación del documento ante la OMI generó una gran publicidad en el medio marítimo. Véase Tradewinds, 25 de julio de 2003 y Lloyd's List, 16 de julio de 2003, entre otros.

37 Entre éstos destacan: Aegean Sea, Braer, Sea Empress, Erika, Ievoli Sun y Prestige; así como otros accidentes que estuvieron a punto de sufrir otros buques como el Mimosa y el Princess Eva. 
Al justificar la solicitud en la importancia y vulnerabilidad de la zona marina en cuestión, los solicitantes destacaron que una región tan diversa conlleva una enorme importancia por el elevado número de mamíferos marinos y aves marinas, la migración e invernada de aves, las especies piscícolas (incluidas especies de peces raras y amenazadas), la variedad de hábitats costeros que proporcionan espacios de reproducción para peces, crustáceos y moluscos, alimento para aves; así como diversidad de hábitats para las plantas.

En opinión de los solicitantes, la zona cumple todos los criterios ecológicos para obtener el carácter de ZMES. Además de ellos, se indicó cómo partes de la zona tienen gran importancia económica o son significativas desde el punto de vista turístico o de ocio. Igualmente, se explicó cómo existen lugares dentro de la zona que tienen un significado especial en términos científicos o educativos. Sin embargo, la actividad del transporte marítimo internacional supone un grave riesgo para la zona. Los factores a considerar son el tipo de cargas que se transportan, el estado de los buques y la intensidad del tráfico, combinados con las condiciones naturales, de carácter hidrográfico, oceanográfico y meteorológico. Las medidas de protección, originalmente solicitadas — para entrar en vigor en julio de 2004 - consistieron en los siguientes elementos:

a) Prohibición del transporte de hidrocarburos pesados por la ZMES en buques de más de 600 toneladas de peso muerto, a no ser buques tanque de doble casco, mismos que se verán obligados a notificarse con 48 horas de antelación. Lo anterior, en el entendido de que los hidrocarburos pesados se definen de la siguiente forma:

i) petróleos pesados, son los que tienen una densidad superior a $900 \mathrm{~kg} / \mathrm{m} 3$ a $15^{\circ} \mathrm{C}$ (lo que significa un grado API inferior a 25.7); ii) combustibles pesados, son los que tienen una densidad superior a $900 \mathrm{~kg} / \mathrm{m} 3$ a $15^{\circ} \mathrm{C}$ o una viscosidad cinemática superior a $180 \mathrm{~mm} 2 / \mathrm{s}$ a $50^{\circ} \mathrm{C}$; y iii) asfalto, alquitrán y emulsiones. Para todos los buques tanque entre 600 y 5000 toneladas de peso muerto, la disposición fue solicitada para ser aplicada a partir de 2008.

b) En concordancia con las Directrices, los solicitantes se reservaron su derecho para proponer otras medidas asociadas a las anteriores, duran- 
te el transcurso del procedimiento de determinación de la ZMES en cuestión. ${ }^{38}$

Antes de destacar el interesante debate que en su momento generó la propuesta de la ZMES de Europa Occidental, es conveniente tener en consideración el vigoroso apoyo por parte del Fondo Mundial para la Naturaleza (FMN, más conocido por sus siglas en inglés WWF) el cual manifestó además que otras zonas marinas contiguas, como el Mar de Irlanda, las costas orientales de Escocia y de Inglaterra hasta East Anglia también cumplen los criterios para ser ZMES, y por tanto debían incluirse en la propuesta. Más aún, el WWF señaló la insuficiencia de la sola medida de prohibición de los cascos sencillos, y manifestó su convicción de que las medidas de protección deberían de ser más estrictas a lo solicitado por los países europeos.

\section{El debate sobre la validez}

El interesante debate sobre la validez de la ZMES de Europa Occidental llevado a cabo durante la sesión 49a. del MEPC en abril de 2003 es susceptible de ser analizado en tres momentos: a) el posicionamiento inicial; b) las negociaciones del grupo oficioso; y c) la decisión del MEPC. En el fondo, la discusión se polarizó entre los proponentes por una parte, y tres segmentos de países que se consideraban perjudicados por distintas razones cada uno.

El primer segmento de críticos a la solicitud de la ZMES se compuso por los Estados representantes de la industria naviera a nivel global; esto es los principales registros abiertos: Panamá y Liberia. El segundo grupo, comandado por Noruega, si bien no representaba a sus vecinos nórdicos, sí expresaba las preocupaciones ambientales que la nueva ZMES traería para sus costas. Por último, el tercer grupo -igualmente sin representación expresa - abanderaba la causa comercial regional consistente en el costo excesivo para los buques tanque para poder realizar tráficos desde y hacia sus puertos, en caso de aprobarse la ZMES. ${ }^{39}$

Así pues, teniendo esta primera visión de conjunto, tanto de las etapas del debate como de los interlocutores, a continuación se señalarán los

39 El principal documento de referencia para profundizar en el debate es el MEPC 49/22. 
tópicos más relevantes en la discusión que tuvo por resultado la aprobación en principio de la ZMES de Europa Occidental.

a) La preocupación sobre las grandes dimensiones de la zona propuesta, y la posibilidad de que se cree un precedente para determinar como ZMES, otras zonas de las mismas dimensiones. ${ }^{40}$

b) La inquietud de que la propuesta creara un precedente que comprometiera el paso inocente y la libertad de navegación, así como la posible violación del derecho internacional al prohibir el paso de buques de casco sencillo por estrechos internacionales.

c) Las potenciales consecuencias adversas que las medidas de protección propuestas tendrían para la seguridad de la navegación, dado que los buques tanque de casco sencillo se verían obligados a navegar por aguas más alejadas de la costa y peligrosas. ${ }^{41}$

d) La duda sobre el fundamento jurídico de las medidas de protección propuestas.

Además de las cuatro preocupaciones básicas que cuestionaron la validez y conveniencia de la ZMES solicitada, se pusieron de manifiesto otras inquietudes, como la falta de claridad sobre la forma en que se aplicarán las medidas de protección; las repercusiones que las medidas de protección propuestas podrían tener sobre el arribo de embarcaciones a puertos de refugio; y por último, la definición de doble casco.

A partir de todas estas observaciones y críticas a la propuesta, el MEPC decidió formar un grupo técnico oficioso con el mandato de investigar si la propuesta cumplía o no con los criterios marcados por las Directrices. El grupo de trabajo no llegó a un consenso generalizado para apoyar la propuesta, pero sí en cambio logró un cambio en la posición de los solicitantes, consistentes en que éstos retiraron la porción de la propuesta relativa a la prohibición de los buques tanque de casco sencillo. ${ }^{42}$ En todo caso, uno de los temas de acuerdo fue la necesidad de recomen-

40 Sobre este punto, la réplica de los solicitantes consistió en señalar que ya se cuenta con precedentes como el de la Gran Barrera de Coral.

41 Noruega señaló que las medidas de protección correspondientes tendrían como consecuencia que los petroleros de casco sencillo que transporten hidrocarburos pesados desde la región del Báltico navegarán más cerca de las costas de Noruega.

42 Sin embargo, para la industria y los grandes registros abiertos como Panamá y Liberia fue motivo de sospecha el retiro de dicha porción de la propuesta, ya que sin ella se debe cuestionar la designación de la región como ZMES. Estas delegaciones han manifestado su preocupación por las posibles medidas de protección que pretendan adoptarse en el futuro. Así pues, si bien el retiro de tal porción de la propuesta generó una mayor simpatía, permanece el escepticismo por los críticos de la misma. Véase LEG 87/16/1. 
dar la revisión de las Directrices con el fin de garantizar la adecuada designación de ZMES en el futuro.

Fue de esta manera como, por apoyo mayoritario, el MEPC aprobó en principio la designación de una ZMES en las aguas de Europa Occidental, con la salvedad de que la zona se redujera, de modo que su límite oriental a la altura de las islas Shetland se situara en la longitud $0^{\circ}{ }^{43} \mathrm{Sin}$ embargo, ante la persistencia de la Federación Rusa de que el Comité Jurídico analizara la propuesta y rindiera un informe con antelación a la sesión del MEPC de octubre de 2004, en donde habría de aprobarse en definitiva la designación de la ZMES de Europa Occidental, se acordó que las delegaciones interesadas harían llegar sus observaciones de índole jurídica a dicho comité para que el MEPC estuviera en condiciones suficientes para tomar una decisión definitiva.

Al quedar aprobada en principio la designación de la ZMES de Europa Occidental en el seno del MEPC de abril de 2003, y en el entendido que la designación definitiva no sería sino hasta octubre de 2004, la industria naviera, los principales países de libre registro y algunas otras naciones afectadas, como la Federación Rusa, decidieron llevar la batalla - hasta entonces perdida para ellos - a otro frente: el del Comité Jurídico. ${ }^{44}$ En él, plantearon una vez más sus consideraciones legales. En esencia, los planteamientos fueron los siguientes:

a) Si bien, al desarrollar las normas relativas a zonas necesitadas de especial protección ambiental, la Convemar tenía presentes a regiones como la de la Gran Barrera de Coral, no se pensaba en zonas tan amplias y diversas como las solicitadas por Europa Occidental, ya que ésta no consiste en un ecosistema único y claramente definido. Así, la designación de una región tan extensa y diversa se apartaría del concepto propio de una ZMES, erosionando la importancia de las actuales ZMES y poniendo en cuestión toda razón de ser de un mecanismo tan importante como la prevención de la contaminación.

43 De conformidad con las Directrices, se acordó igualmente la remisión al Subcomité NAV para analizar las medidas de notificación obligatoria de 48 horas, con el fin de contar en octubre de 2004, con los elementos suficientes para aprobar en definitiva la designación de la ZMES solicitada y sus respectivas medidas de protección.

44 Los argumentos jurídicos fueron presentados por Liberia, Panamá, la Federación Rusa, BIMCO, ICS, INTERCARGO, INTERTANKO e IPTA a la sesión del Comité Jurídico de abril de 2004. Se trata del documento LEG 87/16/1. 
b) La Convemar faculta a los Estados ribereños a adoptar medidas excepcionales mediante las que se restrinja la navegación en zonas que exijan una protección especial. No obstante, estas medidas excepcionales han de estar bien fundadas y han de ser examinadas cuidadosamente, dado que representan una desviación de las normas generales de la convención. Así, en opinión de las delegaciones aquí referidas, si se designase una zona tan amplia y diversa de un océano como la ZMES, y fuesen de aplicación medidas de protección consistentes en restricciones a la navegación, se corre el peligro de que la excepción se convierta en la regla y se erosionen los principios fundamentales de la Convemar.

c) Se pone en cuestión el objetivo y efectos de la medida de protección propuesta consistente en el requisito de notificar con un periodo de antelación de 48 horas. La medida propuesta podría resultar en la detención de buques, con lo que se les impediría ejercer la libertad de navegación y el derecho de paso inocente.

d) Se estima que en lugar de la designación de la ZMES de Europa Occidental, la estrategia adecuada sería la adopción de medidas más estrictas de organización del tráfico; con ésta se enfrentarían las preocupaciones sobre la contaminación de la región de un modo efectivo. Tales medidas debían centrarse en áreas donde, de conformidad con los precedentes, existe un riesgo real, y donde una evaluación de los riesgos, que haya concluido entre otras cosas con un estudio de la intensidad del tráfico, indique que es necesario la adopción de las mismas. De igual forma, debería también de examinarse la conveniencia de designar como ZMES áreas más pequeñas que acojan ecosistemas únicos que se estime están expuestos a riesgos, junto con la adopción de medidas de protección como la designación de "zonas a evitar".

e) En conclusión, el grupo de países y organizaciones que hemos indicado, solicitó una opinión del Comité Jurídico sobre: a) si la designación de una ZMES en Europa Occidental, tal como la propuesta, es conforme con la Convemar; y b) si la medida de protección consiste en una notificación con 48 horas de antelación, así como cualquier otra medida de protección que pudiese proporcionarse en relación con la antedicha ZMES, está en conformidad con las disposiciones de la Convemar, en especial con las disposiciones relativas a la libertad de navegación, tránsito por estrechos internacionales y paso inocente.

Para el estudio y emisión de una opinión, el Comité Jurídico tomó en consideración tanto la solicitud de análisis legal sobre la validez de la 
ZMES en cuestión como las observaciones sobre ello enviadas por la División de Asuntos Oceánicos y del Derecho del Mar de las Naciones Unidas (DOALOS) acerca de la relación entre la designación de las ZMES y la Convemar; en particular sobre su artículo 2116 ). Debe considerarse que si bien las observaciones de la DOALOS no son vinculantes, lo cierto es que ésta tiene una autoridad relevante para el Comité Jurídico, y en general para la OMI y sus Estados miembros.

En realidad, el Comité Jurídico se limitó a describir las diversas posiciones con respecto a los posicionamientos legales y a destacar las observaciones de la DOALOS sobre la validez de la determinación de la ZMES de Europa Occidental. Luego de un análisis sobre la coherencia entre la designación de la ZMES y la Convemar, del dictamen de la DOALOS se pueden destacar los siguientes razonamientos y conclusiones:

a) Si bien las Directrices para la designación de ZMES se basan en las disposiciones del artículo 211 6) de Convemar, éstas son pormenorizadas, y en las mismas se ha adoptado un enfoque más flexible que concuerda con una comprensión científica más sofisticada y general de los peligros que plantean los buques para el medio marino, lo mismo que con una gama de medidas de protección disponibles en el marco de las competencias de la OMI más amplia de la que se contaba cuando se negociaba la Convemar en la década de los setenta. La legalidad de las Directrices para la designación de ZMES no ha sido puesta en tela de juicio, dado que entra en el marco de las competencias de la OMI sobre la reglamentación de las actividades del transporte marítimo internacional y sus posibles consecuencias para el medio marino. Además, las Directrices se ajustan a lo prescrito en el artículo 237 de la convención, ya que son un acuerdo adoptado posteriormente por la Asamblea de la OMI mediante el que se desarrollan los principios generales establecidos en la convención, en particular los de la parte XII.

b) La propuesta de designación vino acompañada de suficiente información científica pormenorizada sobre las condiciones oceanográficas y ecológicas de la zona, lo mismo que sobre los recursos naturales que deben ser protegidos. Además, se incluyeron datos que demuestran que el tráfico marítimo en la zona es extremadamente denso, que muchos buques transportan hidrocarburos pesados, y que se han producido numerosos accidentes con graves consecuencias para el ambiente, incluidos los provocados por los buques Aegean Sea, Erika y Prestige. 
c) En lo que atañe a la superficie de la zona, el artículo 211 6) solamente dispone que ésta ha de ser "un área particular y claramente definida de sus respectivas zonas económicas exclusivas". Si bien del texto se puede deducir, en principio, que el área no debería abarcar todas las zonas económicas exclusivas, no se establece una restricción máxima en cuanto a su extensión.

d) Si bien es cierto que la ZMES de Europa Occidental abarca diversos ecosistemas, en el artículo 2116 ) no hay ninguna disposición que especifique que la zona en cuestión sólo deba abarcar un ecosistema. Así, podrían estar incluidos varios, siempre que todos sean vulnerables a la contaminación provocada por el tráfico marítimo.

e) Con relación a las medidas de protección propuestas, se aclara que el artículo 211 no prohíbe la adopción de exigencias en materia de notificación. Así, ha de ser la OMI la que determine el tipo de medidas de protección aplicables. Por otra parte, no existe ninguna disposición en el Convenio SOLAS relativa a una notificación con un periodo de antelación de 48 horas. Si bien se ha manifestado la preocupación de que el requisito de las 48 horas se aplique como base para prohibir la entrada a la ZMES de Europa Occidental, en infracción del principio de la libertad de navegación, si el Subcomité NAV aprueba esta medida, se estaría también en conformidad con la Convemar, dado que ésta remite a la OMI en lo que atañe a reglas, reglamentos y estándares de navegación.

f) En conclusión, por las razones expuestas, la DOALOS estimó que la solicitud presentada por seis países miembros de la OMI para la designación como ZMES de una zona marina de Europa Occidental, no contraviene la disposiciones de la Convemar. ${ }^{45}$

\section{CONCLUSIONES. HACIA UNA REVISIÓN DE LAS DIRECTRICES SOBRE ZMES}

Si bien es cierto que estamos lejos de un debate como el del siglo XVII entre las posiciones en torno el mare liberum de Grocio y el mare clausum de Seldein, lo es también que la ZMES de Europa Occidental ha

45 En sus conclusiones, la DOALOS tomó en cuenta la limitación en la propuesta original de la ZMES derivada del grupo de trabajo del MEPC de abril de 2003, así como la leve reducción de la misma solicitada a su vez por Noruega. 
generado una preocupación sin precedentes en la industria naviera y en los países reguladores de registros de conveniencia frente a la propuesta de seis países europeos. Así, libertad de navegación, tránsito por estrechos internacionales y paso inocente se autolimitan a la luz de la efectiva protección internacional del medio marino para evitar terribles daños ambientales, como los causados a raíz de los accidentes de buques tanque como el Aegean Sea, el Erika, o el Prestige en las costas del continente europeo.

Si bien el inicio de esta nueva tentativa de "batalla libresca" ha tenido un alto con la opinión jurídica del Comité Jurídico de la Organización Marítima Internacional y de la División de Asuntos Oceánicos y del Derecho del Mar de las Naciones Unidas, mediante la confirmación de que ni las dimensiones geográficas, ni los ecosistemas variados, ni las medidas de protección propuestas para la nueva ZMES representan por sí mismos, violaciones a la Convemar, debe considerarse que la lucha por encontrar un equilibrio entre libertad de navegación y ambiente marino no ha terminado todavía.

En efecto, las voces que se han levantado para pedir una revisión de las Directrices para la designación de ZMES ha llevado al MEPC de la OMI en abril de 2004 ha generar un nuevo debate sobre los contenidos a ser enmendados. Entre los aspectos objeto de revisión aludidos por diversas delegaciones, se encuentran: a) el deber por el Estado solicitante de explicar claramente por qué estima que la zona marina en cuestión no está apropiadamente protegida por las medidas existentes en virtud del Convenio MARPOL y por las medidas de organización del tráfico marítimo; b) el deber de aportar pruebas de que se han adoptado todas las medidas disponibles y que tras un periodo razonable de evaluación, éstas aún no bastan; c) el deber de tomar en cuenta las medidas adoptadas por los Estados ribereños pertinentes para reducir la contaminación procedente de las industrias basadas en tierra, así como la contaminación procedente de la industria establecida en el mar, ya sea debido al transporte marítimo internacional, la explotación mar adentro, las fuentes de energía mar adentro, los ejercicios militares, las actividades pesqueras o el sector de la recreación.

Con esto, es evidente que si bien el debate ideológico del siglo XVII ha quedado atrás, la necesidad de conservación ambiental en balance con los intereses comerciales de la industria naviera son asignaturas contem- 
poráneas y en profundo cambio. Es responsabilidad de todos que la discusión por tal equilibrio tenga en cuenta las diversas perspectivas desde las cuales es necesario abordar el problema, de cara a un futuro sustentable de todas las actividades realizables en los océanos de nuestro planeta. 